\title{
Faithful Translations between Polyvariant Flows and Polymorphic Types
}

\author{
Torben Amtoft ${ }^{1}$ and Franklyn Turbak ${ }^{2}$ * \\ 1 Boston University, Boston MA 02215, USA \\ tamtoft@bu.edu \\ 2 Wellesley College, Wellesley MA 02481, USA \\ fturbak@wellesley.edu
}

\begin{abstract}
Recent work has shown equivalences between various type systems and flow logics. Ideally, the translations upon which such equivalences are based should be faithful in the sense that information is not lost in round-trip translations from flows to types and back or from types to flows and back. Building on the work of Nielson \& Nielson and of Palsberg \& Pavlopoulou, we present the first faithful translations between a class of finitary polyvariant flow analyses and a type system supporting polymorphism in the form of intersection and union types. Additionally, our flow/type correspondence solves several open problems posed by Palsberg \& Pavlopoulou: (1) it expresses call-string based polyvariance (such as k-CFA) as well as argument based polyvariance; (2) it enjoys a subject reduction property for flows as well as for types; and (3) it supports a flow-oriented perspective rather than a type-oriented one.
\end{abstract}

\section{Introduction}

Type systems and flow logic are two popular frameworks for specifying program analyses. While these frameworks seem rather different on the surface, both describe the "plumbing" of a program, and recent work has uncovered deep connections between them. For example, Palsberg and O'Keefe PO95 demonstrated an equivalence between determining flow safety in the monovariant 0-CFA flow analysis and typability in a system with recursive types and subtyping AC93. Heintze showed equivalences between four restrictions of 0-CFA and four type systems parameterized by (1) subtyping and (2) recursive types [Hei95].

Because they merge flow information for all calls to a function, monovariant analyses are imprecise. Greater precision can be obtained via polyvariant analyses, in which functions can be analyzed in multiple abstract contexts. Examples of polyvariant analyses include call-string based approaches, such as $k$-CFA Shi91JW95 NN97, polymorphic splitting WJ98, type-directed flow analysis JWW97, and argument based polyvariance, such as Schmidt's analysis [Sch95] and Agesen's cartesian product analysis Age95. In terms of the

* Both authors were supported by NSF grant EIA-9806747. This work was conducted as part of the Church Project (http://www.cs.bu.edu/groups/church/). 
flow/type correspondence, several forms of flow polyvariance appear to correspond to type polymorphism expressed with intersection and union types Ban97 WDMT97DMTW97PP99. Intuitively, intersection types are finitary polymorphic types that model the multiple analyses for a given abstract closure, while union types are finitary existential types that model the merging of abstract values where flow paths join. Palsberg and Pavlopoulou (henceforth P\&P) were the first to formalize this correspondence by demonstrating an equivalence between a class of flow analyses supporting argument based polyvariance and a type system with union and intersection types [PP99].

If type and flow systems encode similar information, translations between the two should be faithful, in the sense that round-trip translations from flow analyses to type derivations and back (or from type derivations to flow analyses and back) should not lose precision. Faithfulness formalizes the intuitive notion that a flow analysis and its corresponding type derivation contain the same information content. Interestingly, neither the translations of Palsberg and O'Keefe nor those of $\mathrm{P} \& \mathrm{P}$ are faithful. The lack of faithfulness in $\mathrm{P} \& \mathrm{P}$ is demonstrated by a simple example. Let $e=\left(\lambda^{1} \mathrm{x}\right.$. succ $\left.\mathrm{x}\right) @\left(\left(\lambda^{2} \mathrm{y} \cdot \mathrm{y}\right) @ 3\right)$, where we have labeled two program points of interest. Consider an initial monovariant flow analysis in which the only abstract closure reaching point 1 is $v_{1}=(\lambda \mathrm{x}$.succ $\mathrm{x},[])$ and the only one reaching point 2 is $v_{2}=(\lambda \mathrm{y} \cdot \mathrm{y},[])$. The flow-to-type translation of $\mathrm{P} \& \mathrm{P}$ yields the expected type derivation:

$$
\frac{\cdots}{[] \vdash \lambda^{1} x \cdot \operatorname{succ} x: \text { int } \rightarrow \text { int }} \frac{\overline{[] \vdash \lambda^{2} y \cdot y: \text { int } \rightarrow \text { int }} \cdots}{[] \vdash\left(\lambda^{2} y \cdot y\right) @ 3: \text { int }}
$$

However, P\&P's type-to-flow translation loses precision by merging into a single set all abstract closures associated with the same type in a given derivation. For the example derivation above, the type int $\rightarrow$ int translates back to the abstract closure set $V=\left\{v_{1}, v_{2}\right\}$, yielding a less precise flow analysis in which $V$ flows to both points 1 and 2. In contrast, Heintze's translations are faithful. The undesirable merging in the above example is avoided by annotating function types with a label set indicating the source point of the function value. Thus, $\lambda^{1} \mathrm{x}$. succ $\mathrm{x}$ has type int $\stackrel{\{1\}}{\rightarrow}$ int while $\lambda^{2} \mathrm{y} \cdot \mathrm{y}$ has type int $\stackrel{\{2\}}{\rightarrow}$ int.

In this paper, we present the first faithful translations between a broad class of polyvariant flow analyses and a type system with polymorphism in the form of intersection and union types. The translations are faithful in the sense that a round-trip translation acts as the identity for canonical types/flows, and otherwise canonicalizes. In particular, our round-trip translation for types preserves non-recursive types that $\mathrm{P} \& \mathrm{P}$ may transform to recursive types. We achieve this result by adapting the translations of $\mathrm{P} \& \mathrm{P}$ to use a modified version of the flow analysis framework of Nielson and Nielson (henceforth N\&N) [NN97]. As in Heintze's translations, annotations play a key role in the faithfulness of our translations: we (1) annotate flow values to indicate the sinks to which they flow, and (2) annotate union and intersection types with component labels. These annotations can be justified independently of the flow/type correspondence. 
Additionally, our framework solves several open problems posed by P\&P:

1. Unifying $P \mathscr{E} P$ and $N \mathscr{E} N$ : Whereas $\mathrm{P} \& \mathrm{P}$ 's flow specification can readily handle only argument based polyvariance, N\&N's flow specification can also express call-string based polyvariance. So our translations give the first type system corresponding to $k$-CFA analysis where $k \geq 1$.

2. Subject reduction for flows: We inherit from N\&N's flow logic the property that flow information valid before a reduction step is still valid afterwards. In contrast, $\mathrm{P} \& \mathrm{P}$ 's flow system does not have this property.

3. Letting "flows have their way": $\mathrm{P} \& \mathrm{P}$ discuss mismatches between flow and type systems that imply the need to choose one perspective over the other when designing a translation between the two systems. P\&P always let types "have their way"; for example they require analyses to be finitary and to analyze all closure bodies, even though they may be dead code. In contrast, our design also lets flows "have their way", in that our type system does not require all subexpressions to be analyzed.

Due to space limitations, the following presentation is necessarily somewhat dense. Please see the companion technical report AT00 for a more detailed exposition with additional explanatory text, more examples, and proofs.

\section{The Language}

We consider a language whose core is $\lambda$-calculus with recursion:

$$
\begin{aligned}
& u e \in \mathbf{U n L a b E x p r}::=z|\mu f . \lambda x . e| e @ e|c| \operatorname{succ} e \mid \text { ifo } e \text { then } e \text { else } e \mid \ldots \\
& e \in \mathbf{L a b E x p r}::=u e^{l} \quad l \in \mathbf{L a b} \quad z \in \mathbf{V a r}::=x \mid f \quad x \in \mathbf{N V a r} \quad f \in \mathbf{R V a r}
\end{aligned}
$$

$\mu f . \lambda x . e$ denotes a function with parameter $x$ which may call itself via $f ; \lambda x . e$ is a shorthand for $\mu f . \lambda x . e$ where $f$ does not occur in $e$. Recursive variables (ranged over by $f$ ) and non-recursive variables (ranged over by $x$ ) are distinct; $z$ ranges over both. There are also integer constants $c$, the successor function, and the ability to test for zero. Other constructs might be added, e.g., let1.

All subexpressions have integer labels. We often write labels on constructors (e.g., write $\lambda^{l}$ x.e for $(\lambda x . e)^{l}$ and $e_{1} @_{l} e_{2}$ for $\left.\left(e_{1} @ e_{2}\right)^{l}\right)$.

Example 1. The expression $\mathrm{P}_{1} \equiv\left(\lambda^{6} \mathrm{~g} \cdot\left(\left(\mathrm{g}^{3} @_{2} \mathrm{~g}^{4}\right) @_{1} 0^{5}\right)\right) @_{0}\left(\lambda^{8} \mathrm{x} \cdot \mathrm{x}^{7}\right)$ shows the need for polyvariance: $\lambda^{8} \mathrm{x} \cdot \mathrm{x}^{7}$ is applied both to itself and to an integer.

Like N\&N, but unlike P\&P, we use an environment-based small step semantics. This requires incorporating N\&N's bind and close constructs into our expression syntax. An expression not containing bind or close is said to be pure. Every abstraction body must be pure. A program $P$ is a pure, closed expression where each label occurs at most once within $P$; thus each subexpression of $P(\in$ $\left.\operatorname{SubExp}_{P}\right)$ denotes a unique "position" within $P$.

\footnotetext{
${ }^{1}$ Let-polymorphism can be simulated by intersection types.
} 


\section{The Type System}

Types are built from base types, function types, intersection types, and union types as follows (where ITag and UTag are unspecified):

$$
\begin{aligned}
& t \in \text { ElementaryType }::=\text { int } \mid \bigwedge_{i \in I}\left\{K_{i}: u_{i} \rightarrow u_{i}^{\prime}\right\} \\
& u \in \text { UnionType }::=\bigvee_{i \in I}\left\{q_{i}: t_{i}\right\} \\
& K \in \mathcal{P}(\text { ITag }) \quad k \in \text { ITag } \quad q \in \text { UTag }
\end{aligned}
$$

Such grammars are usually interpreted inductively, but this one is to be viewed co-inductively. That is, types are regular (possibly infinite) trees formed according to the above specification. Two types are considered equal if their infinite unwindings are equal (modulo renaming of the index sets $I$ ).

An elementary type $t$ is either an integer int or an intersection type of the form $\bigwedge_{i \in I}\left\{K_{i}: u_{i} \rightarrow u_{i}^{\prime}\right\}$, where $I$ is a (possibly empty) finite index set, each $u_{i}$ and $u_{i}^{\prime}$ is a union type, and the $K_{i}$ 's, known as I-tagsets, are non-empty disjoint sets of $I$-tags. We write $d o m(t)$ for $\cup_{i \in I} K_{i}$. Intuitively, if an expression $e$ has the above intersection type then for all $i \in I$ it holds that the expression maps values of type $u_{i}$ into values of type $u_{i}^{\prime}$.

A union type $u$ has the form $\bigvee_{i \in I}\left\{q_{i}: t_{i}\right\}$, where $I$ is a (possibly empty) finite index set, each $t_{i}$ is an elementary type, and the $q_{i}$ are distinct $U$-tags. We write $\operatorname{dom}(u)$ for $\cup_{i \in I}\left\{q_{i}\right\}$, and $u . q=t$ if there exists $i \in I$ such that $q=q_{i}$ and $t=t_{i}$. We assume that for all $i \in I$ it holds that $t_{i}=$ int iff $q_{i}=q_{\text {int }}$ where $q_{\text {int }}$ is a distinguished U-tag. Intuitively, if an expression $e$ has the above union type then there exists an $i \in I$ such that $e$ has the elementary type $t_{i}$.

If $I=\{1 \cdots n\}(n \geq 0)$, we write $\bigvee\left(q_{1}: t_{1}, \cdots, q_{n}: t_{n}\right)$ for $\bigvee_{i \in I}\left\{t_{i}: q_{i}\right\}$ and write $\bigwedge\left(K_{1}: u_{1} \rightarrow u_{1}^{\prime}, \cdots, K_{n}: u_{n} \rightarrow u_{n}^{\prime}\right)$ for $\bigwedge_{i \in I}\left\{K_{i}: u_{i} \rightarrow u_{i}^{\prime}\right\}$. We write $u_{\text {int }}$ for $\bigvee\left(q_{\text {int }}\right.$ : int $)$.

The type system is much as in $\mathrm{P} \& \mathrm{P}$ except for the presence of tags. These annotations serve as witnesses for existentials in the subtyping relation and play crucial roles in the faithfulness of our flow/type correspondence. U-tags track the "source" of each intersection type and help to avoid the precision-losing merging seen in P\&P's type-to-flow translation (cf. Sect. 1). I-tagsets track the "sinks" of each arrow type and help to avoid unnecessary recursive types in the flow-to-type translation.

\subsection{Subtyping}

We define an ordering $\leq$ on union types and an ordering $\leq_{\wedge}$ on elementary types, where $u \leq u^{\prime}$ means that $u^{\prime}$ is less precise than $u$ and similarly for $\leq_{\wedge}$. To capture the intuition that something of type $t_{1}$ has one of the types $t_{1}$ or $t_{2}$, $\leq$ should satisfy $\bigvee\left(q_{1}: t_{1}\right) \leq \bigvee\left(q_{1}: t_{1}, q_{2}: t_{2}\right)$. For $\leq_{\wedge}$, we want to capture the following intuition: a function that can be assigned both types $u_{1} \rightarrow u_{1}^{\prime}$ and $u_{2} \rightarrow u_{2}^{\prime}$ also (1) can be assigned one of them ${ }^{2}$ and (2) can be assigned a function type

$\overline{{ }^{2} \text { I.e., for } i \in}\{1,2\}, \bigwedge\left(K_{1}: u_{1} \rightarrow u_{1}^{\prime}, K_{2}: u_{2} \rightarrow u_{2}^{\prime}\right) \leq \wedge \bigwedge\left(K_{i}: u_{i} \rightarrow u_{i}^{\prime}\right)$. 
that "covers" both 3 . The following mutually recursive specification of $\leq$ and $\leq_{\wedge}$ formalizes these considerations:

$$
\bigvee_{i \in I}\left\{q_{i}: t_{i}\right\} \leq \bigvee_{j \in J}\left\{q_{j}^{\prime}: t_{j}^{\prime}\right\}
$$

iff for all $i \in I$ there exists $j \in J$ such that $q_{i}=q_{j}^{\prime}$ and $t_{i} \leq_{\wedge} t_{j}^{\prime}$

int $\leq_{\wedge}$ int

$$
\bigwedge_{i \in I}\left\{K_{i}: u_{i} \rightarrow u_{i}^{\prime}\right\} \leq \wedge \bigwedge_{j \in J}\left\{K_{j}^{\prime}: u_{j}^{\prime \prime} \rightarrow u_{j}^{\prime \prime \prime}\right\}
$$

iff for all $j \in J$ there exists $I_{0} \subseteq I$ such that

$$
\begin{aligned}
& K_{j}^{\prime}=\cup_{i \in I_{0}} K_{i} \text { and } \forall i \in I_{0} . u_{i}^{\prime} \leq u_{j}^{\prime \prime \prime} \text { and } \\
& \forall q \in \operatorname{dom}\left(u_{j}^{\prime \prime}\right) . \exists i \in I_{0} . q \in \operatorname{dom}\left(u_{i}\right) \text { and } u_{j}^{\prime \prime} . q \leq_{\wedge} u_{i} . q .
\end{aligned}
$$

Observe that if $t \leq_{\wedge} t^{\prime}$, then $\operatorname{dom}\left(t^{\prime}\right) \subseteq \operatorname{dom}(t)$. The above specification is not yet a definition of $\leq$ and $\leq_{\wedge}$, since types may be infinite. However, it gives rise to a monotone functional on a complete lattice whose elements are pairs of relations; $\leq$ and $\leq_{\wedge}$ are then defined as the (components of) the greatest fixed point of this functional. Coinduction yields:

Lemma 1. The relations $\leq$ and $\leq_{\wedge}$ are reflexive and transitive.

Our subtyping relation differs from P\&P's in several ways. The U-tags and I-tags serve as "witnesses" for the existential quantifiers present in the specification, reducing the need for search during type checking. Finally, our $\leq$ seems more natural that the $\mathrm{P} \& \mathrm{P}$ 's $\leq_{1}$, which is not a congruence and in fact has the rather odd property that if $\vee\left(T_{1}, T_{2}\right) \leq_{1} \vee\left(T_{3}, T_{4}\right)$ (with the $T_{i}$ 's all distinct), then either $\vee\left(T_{1}, T_{2}\right) \leq_{1} T_{3}$ or $\vee\left(T_{1}, T_{2}\right) \leq_{1} T_{4}$.

\subsection{Typing Rules}

A typing $T$ for a program $P$ is a tuple $\left(P, I T_{T}, U T_{T}, D_{T}\right)$, where $I T_{T}$ is a finite set of I-tags, $U T_{T}$ is a finite set of U-tags, and $D_{T}$ is a derivation of [] $\vdash P: u$ according to the inference rules given in Fig. 1. In a judgement $A \vdash e: u, A$ is an environment with bindings of the form $[z \mapsto u]$; we require that all I-tags in $D_{T}$ belong to $I T_{T}$ and that all U-tags in $D_{T}$ belong to $U T_{T}$.

Subtyping has been inlined in all of the rules to simplify the type/flow correspondence. The rules for function abstraction and function application are both instrumented with a "witness" that enables reconstructing the justification for applying the rule. In [app] ${ }^{w^{@}}$, the type of the operator is a (possibly empty) union, all components of which have the expected function type but the I-tagsets may differ; the app-witness $w^{@}$ is a partial mapping from $\operatorname{dom}\left(u_{1}\right)$ that given $q$ produces the corresponding I-tagset. In [fun] ${ }^{w^{\lambda}}$, the function types resulting from analyzing the body in several different environments are combined into an intersection type $t$. This is wrapped into a union type with an arbitrary U-tag $q$,

\footnotetext{
${ }^{3}$ I.e., $\bigwedge\left(K_{1}: u_{1} \rightarrow u_{1}^{\prime}, K_{2}: u_{2} \rightarrow u_{2}^{\prime}\right) \leq \wedge \bigwedge\left(K_{1} \cup K_{2}: u_{12} \rightarrow u_{12}^{\prime}\right)$, where any value having one of the types $u_{1}^{\prime}$ or $u_{2}^{\prime}$ also has type $u_{12}^{\prime}$, and where any value having type $u_{12}$ also has one of the types $u_{1}$ or $u_{2}$.
} 


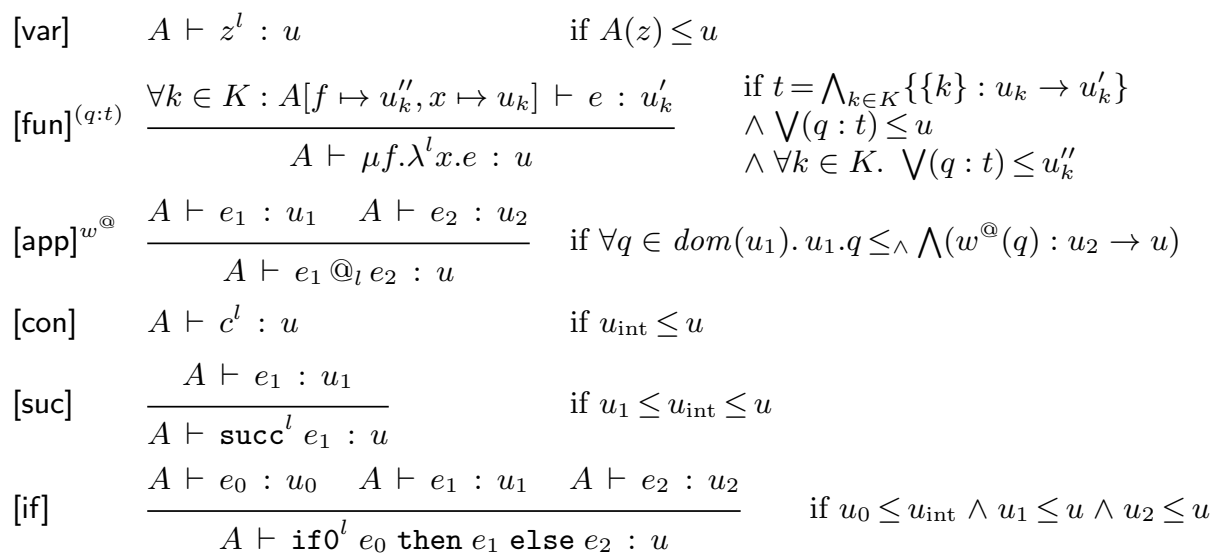

Fig. 1. The typing rules

$$
\begin{aligned}
& A_{\mathrm{g}} \vdash \mathrm{g}^{3}: u_{\mathrm{x}} \quad A_{\mathrm{g}} \vdash \mathrm{g}^{4}: u_{\mathrm{x}}^{\prime} \\
& \frac{A_{\mathrm{g}} \vdash \mathrm{g}^{3} @_{2} \mathrm{~g}^{4}: u_{\mathrm{x}}^{\prime} A_{\mathrm{g}} \vdash 0^{5}: u_{\text {int }}}{\frac{A_{\mathrm{g}} \vdash\left(\mathrm{g}^{3} @_{2} \mathrm{~g}^{4}\right) @_{1} 0^{5}: u_{\text {int }}}{[] \vdash \lambda^{6} \mathrm{~g} \cdot\left(\left(\mathrm{g}^{3} @_{2} \mathrm{~g}^{4}\right) @_{1} 0^{5}\right): u_{\mathrm{g}}}} \frac{A_{\mathrm{x}} \vdash \mathrm{x}^{7}: u_{\text {int }} \quad A_{\mathrm{x}}^{\prime} \vdash \mathrm{x}^{7}: u_{\mathrm{x}}^{\prime}}{[] \vdash\left(\lambda^{6} \mathrm{~g} \cdot\left(\left(\mathrm{g}^{3} @_{2} \mathrm{~g}^{4}\right) @_{1} 0^{5}\right)\right) @_{0}\left(\lambda^{8} \mathrm{x} \cdot \mathrm{x}^{7}\right): u_{\text {int }}}
\end{aligned}
$$

Fig. 2. A derivation $D_{\mathrm{T}_{1}}$ for the program $\mathrm{P}_{1}$ from Example [1

which provides a way of keeping track of the origin of a function type (cf. Sects. 1 and 5). Accordingly, the fun-witness $w^{\lambda}$ of this inference is the pair $(q: t)$. Note that $K$ may be empty in which case the body is not analyzed.

Example 2. For the program $\mathrm{P}_{1}$ from Ex. 1, we can construct a typing $\mathrm{T}_{1}$ as follows: $I T_{\mathrm{T}_{1}}=\{0,1,2\}, U T_{\mathrm{T}_{1}}=\left\{q_{\mathrm{x}}, q_{\mathrm{g}}\right\}$, and $D_{\mathrm{T}_{1}}$ is as in Fig. [2] where

$$
\begin{aligned}
& u_{\mathrm{x}}^{\prime}=\bigvee\left(q_{\mathrm{x}}: \bigwedge\left(\{1\}: u_{\mathrm{int}} \rightarrow u_{\mathrm{int}}\right)\right) \\
& u_{\mathrm{x}}=\bigvee\left(q_{\mathrm{x}}: \bigwedge\left(\{1\}: u_{\mathrm{int}} \rightarrow u_{\mathrm{int}},\{2\}: u_{\mathrm{x}}^{\prime} \rightarrow u_{\mathrm{x}}^{\prime}\right)\right) \\
& u_{\mathrm{g}}=\bigvee\left(q_{\mathrm{g}}: \bigwedge\left(\{0\}: u_{\mathrm{x}} \rightarrow u_{\mathrm{int}}\right)\right) \\
& A_{\mathrm{g}}=\left[\mathrm{g} \mapsto u_{\mathrm{x}}\right] \quad A_{\mathrm{x}}=\left[\mathrm{x} \mapsto u_{\mathrm{int}}\right] \quad A_{\mathrm{x}}^{\prime}=\left[\mathrm{x} \mapsto u_{\mathrm{x}}^{\prime}\right]
\end{aligned}
$$

Note that $u_{\mathrm{x}} \leq u_{\mathrm{x}}^{\prime}$, and that $u_{\mathrm{x}} \cdot q_{\mathrm{x}} \leq \wedge \bigwedge\left(\{2\}: u_{\mathrm{x}}^{\prime} \rightarrow u_{\mathrm{x}}^{\prime}\right)$ so that $\left\{q_{\mathrm{x}} \mapsto\{2\}\right\}$ is indeed an app-witness for the inference at the top left of Fig. 2,

The type system in Fig. 1] can be augmented with rules for bind and close such that the resulting system satisfies a subject reduction property. The soundness of the type system follows from subject reduction, since "stuck" expressions (such as $7 @ 9$ ) are not typable.

In a typing $T$ for $P$, for each $e$ in $\operatorname{SubExpr}_{P}$ there may be several judgements for $e$ in $D_{T}$, due to the multiple analyses performed by [fun]. We assign to each 
judgement $J$ for $e$ in $D_{T}$ an environment ke (its address) that for all applications of [fun] in the path from the root of $D_{T}$ to $J$ associates the bound variables with the branch taken. In $D_{\mathrm{T}_{1}}$ (Fig. 2), the judgement $A_{\mathrm{x}} \vdash \mathrm{x}^{7}: u_{\text {int }}$ has address $[\mathrm{x} \mapsto 1]$ and the judgement $A_{\mathrm{x}}^{\prime} \vdash \mathrm{x}^{7}: u_{\mathrm{x}}^{\prime}$ has address $[\mathrm{x} \mapsto 2]$.

The translation in Sect. [5 requires that a typing must be uniform, i.e., the following partial function $A_{T}$ must be well-defined: $A_{T}(z, k)=u$ iff $D_{T}$ contains a judgement of the form $A \vdash e: u^{\prime}$ with address $k e$, where $k e(z)=k$ and $A(z)=u$. For $\mathrm{T}_{1}$ we have, e.g., $A_{\mathrm{T}_{1}}(\mathrm{x}, 1)=u_{\text {int }}$ and $A_{\mathrm{T}_{1}}(\mathrm{x}, 2)=u_{\mathrm{x}}^{\prime}$.

\section{The Flow System}

Our system for flow analysis has the form of a flow logic, in the style of N\&N. A flow analysis $F$ for program $P$ is a tuple $\left(P, \operatorname{Mem}_{F}, \mathcal{C}_{F}, \rho_{F}, \Phi_{F}\right)$, whose components are explained below (together with some auxiliary derived concepts).

Polyvariance is modeled by mementoes, where a memento $\left(m \in \mathrm{Mem}_{F}\right)$ represents a context for analyzing the body of a function. We shall assume that $M_{e} m_{F}$ is non-empty and finite; then all other entities occurring in $F$ will also be finite. Each expression $e$ is analyzed wrt. several different memento environments, where the entries of a memento environment $\left(m e \in \operatorname{MemEnv}_{F}\right.$ ) take the form $[z \mapsto m]$ with $m$ in $M_{e m}$. Accordingly, a flow configuration $\left(\in\right.$ FlowConf $\left._{F}\right)$ is a pair $(e, m e)$, where $F V(e) \subseteq \operatorname{dom}(m e)$.

The goal of the flow analysis is to associate a set of flow values to each configuration, where a flow value $\left(v \in{\text { Flow } V a l_{F}}_{F}\right)$ is either an integer Int or of the form $(a c, M)$, where $a c\left(\in\right.$ AbsClos $\left._{F}\right)$ is an abstract closure of the form ( $\left.f n, m e\right)$

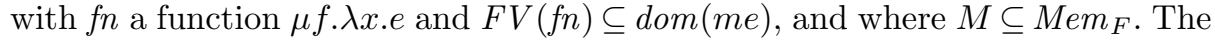
$M$ component can be thought of a superset of the "sinks" of the abstract closure $a c$, i.e. the contexts in which it is going to be applied. Our flow values differ from N\&N's in two respects: (i) they do not include the memento that corresponds to the point of definition; (ii) they do include the mementoes of use (the $M$ component), in order to get a flow system that is almost isomorphic to the type system of Sect. 3. This extension does not make it harder to analyze an expression, since one might just let $M=M e m_{F}$ everywhere.

A flow set $V\left(\in\right.$ FlowSet $\left._{F}\right)$ is a set of flow values, with the property that if $\left(a c, M_{1}\right) \in V$ and $\left(a c, M_{2}\right) \in V$ then $M_{1}=M_{2}$. We define an ordering on FlowSet $F$ by stipulating that $V_{1} \leq_{V} V_{2}$ iff for all $v_{1} \in V_{1}$ there exists $v_{2} \in V_{2}$ such that $v_{1} \leq_{v} v_{2}$, where the ordering $\leq_{v}$ on Flow $V_{F l}$ is defined by stipulating that Int $\leq_{v}$ Int and that $\left(a c, M_{1}\right) \leq_{v}\left(a c, M_{2}\right)$ iff $M_{2} \subseteq M_{1}$. Note that if $V_{1} \leq_{V} V_{2}$ then $V_{2}$ is obtained From $V_{1}$ by adding some "sources" and removing some "sinks (in a sense moving along a "flow path" from a source to a sink), so in that respect the ordering is similar to the type ordering in [WDMT97.

$\Phi_{F}$ is a partial mapping from $\left(\operatorname{Labs}_{P} \times \operatorname{MemEnv}_{F}\right) \times \operatorname{AbsClos}_{F}$ to $\mathcal{P}\left(\mathrm{Mem}_{F}\right)$, where $\operatorname{Labs}_{P}$ is the set of labels occurring in $P$. Intuitively, if the abstract closure $a c$ in the context $m e$ is applied to an expression with label $l$, then $\Phi_{F}((l, m e), a c)$ denotes the actual sinks of $a c$. 
$\mathcal{C}_{F}$ is a mapping from Labs $_{P} \times$ MemEnv $_{F}$ to $(\text { FlowSet } F)_{\perp}$. Intuitively, if $\mathcal{C}_{F}(l, m e)=V(\neq \perp)$ and $\mathcal{C}_{F}$ is valid (defined below) for the flow configuration $\left(u e^{l}, m e\right)$ then all semantic values that $u e^{l}$ may evaluate to in a semantic environment approximated by me can be approximated by the set $V$. Similarly, $\rho_{F}(z, m)$ approximates the set of semantic values to which $z$ may be bound when analyzed in memento $m$.

Unlike $\mathrm{N} \& \mathrm{~N}$, we distinguish between $\mathcal{C}_{F}(l, m e)$ being the empty set and being $\perp$. The latter means that no flow configuration $\left(u e^{l}, m e\right)$ is "reachable", and so there is no need to analyze it. The relation $\leq_{V}$ on FlowSet $F$ is lifted to a relation $\leq_{V}$ on FlowSet ${ }_{F \perp}$.

Example 3. For the program $\mathrm{P}_{1}$ from Ex. 1 a flow analysis $\mathrm{F}_{1}$ with $M_{\mathrm{Nem}} \mathrm{F}_{1}=$ $\{0,1,2\}$ is given below. We have named some entities (note that $v_{\mathrm{x}} \leq_{v} v_{\mathrm{x}}^{\prime}$ ):

$$
\begin{array}{lll}
m e_{\mathrm{g}}=[\mathrm{g} \mapsto 0] & a c_{\mathrm{g}}=(\lambda \mathrm{g} \cdot \cdots,[]) & v_{\mathrm{g}}=\left(a c_{\mathrm{g}},\{0\}\right) \\
m e_{\mathrm{x} 1}=[\mathrm{x} \mapsto 1] & a c_{\mathrm{x}}=\left(\lambda \mathrm{x} \cdot \mathrm{x}^{7},[]\right) & v_{\mathrm{x}}^{\prime}=\left(a c_{\mathrm{x}},\{1\}\right) \\
m e_{\mathrm{x} 2}=[\mathrm{x} \mapsto 2] & & v_{\mathrm{x}}=\left(a c_{\mathrm{x}},\{1,2\}\right)
\end{array}
$$

$\mathcal{C}_{\mathrm{F}_{1}}$ and $\rho_{\mathrm{F}_{1}}$ are given by the entries below (all other are $\perp$ ):

$$
\begin{aligned}
\left\{v_{\mathrm{g}}\right\} & =\mathcal{C}_{\mathrm{F}_{1}}(6,[]) \\
\{\operatorname{Int}\} & =\rho_{\mathrm{F}_{1}}(\mathrm{x}, 1)=\mathcal{C}_{\mathrm{F}_{1}}\left(7, m e_{\mathrm{x} 1}\right)=\mathcal{C}_{\mathrm{F}_{1}}\left(5, m e_{\mathrm{g}}\right)=\mathcal{C}_{\mathrm{F}_{1}}\left(1, m e_{\mathrm{g}}\right)=\mathcal{C}_{\mathrm{F}_{1}}(0,[]) \\
\left\{v_{\mathrm{x}}^{\prime}\right\} & =\rho_{\mathrm{F}_{1}}(\mathrm{x}, 2)=\mathcal{C}_{\mathrm{F}_{1}}\left(7, m e_{\mathrm{x} 2}\right)=\mathcal{C}_{\mathrm{F}_{1}}\left(4, m e_{\mathrm{g}}\right)=\mathcal{C}_{\mathrm{F}_{1}}\left(2, m e_{\mathrm{g}}\right) \\
\left\{v_{\mathrm{x}}\right\} & =\rho_{\mathrm{F}_{1}}(\mathrm{~g}, 0)=\mathcal{C}_{\mathrm{F}_{1}}\left(3, m e_{\mathrm{g}}\right)=\mathcal{C}_{\mathrm{F}_{1}}(8,[])
\end{aligned}
$$

Thus $\left(\mathrm{g}^{3} @_{2} \mathrm{~g}^{4}\right) @_{1} 0^{5}$ is analyzed with $\mathrm{g}$ bound to 0 , and $\mathrm{x}^{7}$ is analyzed twice: with $\mathrm{x}$ bound to 1 and with $\mathrm{x}$ bound to 2 . Accordingly, $\Phi_{\mathrm{F}_{1}}$ is given by

$$
\Phi_{\mathrm{F}_{1}}\left((8,[]), a c_{\mathrm{g}}\right)=\{0\}, \Phi_{\mathrm{F}_{1}}\left(\left(5, m e_{\mathrm{g}}\right), a c_{\mathrm{x}}\right)=\{1\}, \Phi_{\mathrm{F}_{1}}\left(\left(4, m e_{\mathrm{g}}\right), a c_{\mathrm{x}}\right)=\{2\} .
$$

\subsection{Validity}

Of course, not all flow analyses give a correct description of the program being analyzed. To formulate a notion of validity, we define a predicate $F \models^{\text {me }} e$ (to be read: $F$ analyzes $e$ correctly wrt. the memento environment me), with $(e, m e) \in$ Flow Conf $_{F}$. The predicate must satisfy the specification in Fig. 3, which gives rise to a monotone functional on the complete lattice $\mathcal{P}\left(\right.$ Flow Conf $\left._{F}\right)$; following the convincing argument of $\mathrm{N} \& \mathrm{~N}$, we define $F \models^{m e} e$ as the greatest fixed point of this functional so as to be able to cope with recursive functions.

In [fun], we deviate from $\mathrm{N} \& \mathrm{~N}$ by recording me, rather than the restriction of me to $F V\left(\mu f . \lambda x . e_{0}\right)$. As in $\mathrm{P} \& \mathrm{P}$, this facilitates the translations to and from types. In [app], the set $M$ corresponds to P\&P's notion of cover, which in turn is needed to model the "cartesian product" algorithm of Age95. In N\&N's framework, $M$ is always a singleton $\{m\}$; in that case the condition " $\forall v \in$ $\mathcal{C}_{F}\left(l_{2}, m e\right) . \ldots$ " amounts to the simpler " $\mathcal{C}_{F}\left(l_{2}, m e\right) \leq_{V} \rho_{F}(x, m)$ ".

By structural induction in $u e^{l}$ we see that if $F=^{m e} u e^{l}$ then $\mathcal{C}_{F}(l, m e) \neq \perp$. We would also like the converse implication to hold: 


$$
\begin{aligned}
& \text { [var] } \quad F \models{ }^{m e} z^{l} \text { iff } \perp \neq \rho_{F}(z, m e(z)) \leq_{V} \mathcal{C}_{F}(l, m e) \\
& \text { [fun] } \quad F \mid=^{m e} \mu f . \lambda^{l} x . e_{0} \text { iff }\left\{\left(\left(\mu f . \lambda x . e_{0}, m e\right), M e m_{F}\right)\right\} \leq_{V} \mathcal{C}_{F}(l, m e) \\
& {[a p p] \quad F \mid={ }^{m e} u e_{1}{ }^{l_{1}} @_{l} u e_{2}{ }^{l_{2}} \text { iff }} \\
& \mathcal{C}_{F}(l, m e) \neq \perp \wedge F \models \models^{m e} u e_{1}{ }^{l_{1}} \wedge F \models^{m e} u e_{2}{ }^{l_{2}} \wedge \\
& \forall\left(a c_{0}, M_{0}\right) \in \mathcal{C}_{F}\left(l_{1}, m e\right) \\
& \text { let } M=\Phi_{F}\left(\left(l_{2}, m e\right), a c_{0}\right) \text { and }\left(\mu f . \lambda x \cdot u e_{0}{ }^{l_{0}}, m e_{0}\right)=a c_{0} \text { in } \\
& M \subseteq M_{0} \wedge \forall v \in \mathcal{C}_{F}\left(l_{2}, m e\right) . \exists m \in M .\{v\} \leq_{V} \rho_{F}(x, m) \wedge \\
& \forall m \in M: F \models{ }^{m e_{0}[f, x \mapsto m]} u e_{0}{ }^{l_{0}} \wedge \\
& \mathcal{C}_{F}\left(l_{0}, m e_{0}[f, x \mapsto m]\right) \leq_{V} \mathcal{C}_{F}(l, m e) \wedge \\
& \rho_{F}(x, m) \neq \perp \wedge\left\{\left(a c_{0}, \operatorname{Mem}_{F}\right)\right\} \leq_{V} \rho_{F}(f, m)
\end{aligned}
$$

[con] $\quad F \mid{ }^{m e} c^{l}$ iff Int $\in \mathcal{C}_{F}(l, m e)$

[suc] $\quad F \mid=^{m e} \operatorname{succ}^{l} e_{1}$ iff $F \models^{m e} e_{1} \wedge \operatorname{Int} \in \mathcal{C}_{F}(l, m e)$

[if] $\quad F \mid=^{m e}$ if ${ }^{l} e_{0}$ then $u e_{1}{ }^{l_{1}}$ else $u e_{2}{ }^{l_{2}}$ iff

$$
\begin{aligned}
& F \models^{m e} e_{0} \wedge F \models^{m e} u e_{1}{ }^{l_{1}} \wedge F \models^{m e} u e_{2}{ }^{l_{2}} \wedge \\
& \mathcal{C}_{F}\left(l_{1}, m e\right) \leq_{V} \mathcal{C}_{F}(l, m e) \wedge \mathcal{C}_{F}\left(l_{2}, m e\right) \leq_{V} \mathcal{C}_{F}(l, m e)
\end{aligned}
$$

Fig. 3. The flow logic

Definition 1. Let a flow analysis $F$ for $P$ be given. We say that $F$ is valid iff (i) $F \models$ [] $P$; (ii) whenever $e=u e^{l} \in \operatorname{SubExpr}_{P}{\text { with }(e, m e) \in \text { FlowConf }_{F} \text { and }}$ $\mathcal{C}_{F}(l, m e) \neq \perp$ then $F \mid={ }^{m e} e$.

Using techniques as in N\&N, we can augment Fig. 3 with rules for bind and close and then prove a subject reduction property for flows which for closed $E$ reads: if $E$ reduces to $E^{\prime}$ in one evaluation step and $F \models^{[]} E$ then $F \models^{[]} E^{\prime}$.

So far, even for badly behaved programs like $P=7 @ 9$ it is possible (just as in N\&N) to find a $F$ for $P$ such that $F$ is valid. Since our type system rejects such programs, we would like to filter them out:

Definition 2. Let a flow analysis $F$ for $P$ be given. We say that $F$ is safe iff for all ue in SubExpr $_{P}$ and for all me it holds: (i) if $u e=u e_{1}{ }_{1}$ @ $e_{2}$ then Int $\notin \mathcal{C}_{F}\left(l_{1}, m e\right)$; (ii) if ue $=$ succ $u e_{1}{ }_{1}$ then $v \in \mathcal{C}_{F}\left(l_{1}\right.$, me) implies $v=$ Int; (iii) if $u e=$ if $u e_{0}{ }^{l_{0}}$ then $e_{1}$ else $e_{2}$ then $v \in \mathcal{C}_{F}\left(l_{0}\right.$, me) implies $v=$ Int.

Example 4. Referring back to Example 3, it clearly holds that $\mathrm{F}_{1}$ is safe, and it is easy (though a little cumbersome) to verify that $F_{1}$ is valid.

\subsection{Taxonomy of Flow Analyses}

Two common categories of flow analyses are the "call-string based" (e.g., Shi91]) and the "argument-based" (e.g., Sch95 Age95). Our descriptive framework can model both approaches (which can be "mixed", as in [NN99]).

A flow analysis $F$ for $P$ such that $F$ is valid is in CallString $_{\beta}^{P}$, where $\beta$ is a mapping from $\operatorname{Labs}_{P} \times M e m E n v_{F}$ into $\mathrm{Mem}_{F}$, iff whenever $\Phi_{F}\left(\left(l_{2}, m e\right), a c\right)$ is de- 
fined it equals $\{\beta(l, m e)\}$ where $l$ is such that $4 e_{1} @_{l} u e_{2}{ }^{l_{2}} \in \operatorname{SubExpr}_{P}$. All $k$-CFA analyses fit into this category: for $0-\mathrm{CFA}$ we take $\mathrm{Mem}_{F}=\{\bullet\}$ and $\beta(l, m e)=\bullet$; for 1-CFA we take $\operatorname{Mem}_{F}=\operatorname{Labs}_{P}$ and $\beta(l, m e)=l$; and for 2-CFA (the generalization to $k>2$ is immediate) we take $\operatorname{Mem}_{F}=\operatorname{Labs}_{P} \cup\left(\operatorname{Labs}_{P} \times \operatorname{Labs}_{P}\right)$ and define $\beta(l, m e)$ as follows: let it be $l$ if $m e=[]$, and let it be $\left(l, l_{1}\right)$ if $m e$ takes the form $m e^{\prime}[z \mapsto m]$ with $m$ either $l_{1}$ or $\left(l_{1}, l_{2}\right)$.

A flow analysis $F$ for $P$ such that $F$ is valid is in $\operatorname{ArgBased}_{\alpha}^{P}$ iff for all nonrecursive variables $x$ and mementoes $m$ it holds that whenever $\rho_{F}(x, m) \neq \perp$ then $\epsilon_{V}\left(\rho_{F}(x, m)\right)=\alpha(m)$ where $\epsilon_{V}$ removes the $M$ component of a flow value. For this kind of analysis, a memento $m$ essentially denotes a set of abstract closures. To more precisely capture specific argument-based analyses, such as Age95 or the type-directed approach of [JWW97], we may impose further demands on $\alpha$.

Example 5. The flow analysis $\mathrm{F}_{1}$ is a 1-CFA and also in $\operatorname{ArgBased}_{\alpha}{ }^{\mathrm{P}}$, with $\alpha(0)=$ $\alpha(2)=\left\{a c_{\mathrm{x}}\right\}$ and $\alpha(1)=\{$ Int $\}$.

Given a program $P$, it turns out that for all $\beta$ the class CallString $_{\beta}^{P}$, and for certain kinds of $\alpha$ also the class $\operatorname{ArgBased}_{\alpha}^{P}$, contains a least (i.e., most precise) flow analysis; here the ordering on flow analyses is defined pointwise 5 on $\mathcal{C}_{F}, \rho_{F}$ and $\Phi_{F}$. This is much as in N\&N where for all total and deterministic "instantiators" the corresponding class of analyses contains a least element, something we cannot hope for since we allow $\Phi_{F}$ to return a non-singleton.

\subsection{Reachability}

For a flow analysis $F$, some entries may be garbage. To see an example of this, suppose that $\mu f . \lambda x . u e^{l}$ in $\operatorname{SubExpr}_{P}$, and suppose that $\rho_{F}(x, m)=\perp$ for all $m \in M e m_{F}$. From this we infer that the above function is never calle so for all me the value of $\mathcal{C}_{F}(l, m e)$ is uninteresting. It may therefore be replaced by $\perp$, something which is in fact achieved by the roundtrip described in Sect. 7.1.

To formalize a notion of reachability we introduce a set $\operatorname{Reach}_{P}^{F}$ that is intended to encompas: 6 all entries of $\mathcal{C}_{F}$ and $\rho_{F}$ that are "reachable" from the root of $P$. Let Analyzes $F_{m}^{F}\left(\mu f . \lambda x . u e_{0}{ }^{l}, m e\right)$ be a shorthand for $\mathcal{C}_{F}\left(l_{0}, m e[f, x \mapsto m]\right) \neq \perp$ and $\rho_{F}(x, m) \neq \perp$ and $\left\{\left(\left(\mu f . \lambda x . u e_{0}{ }^{l_{0}}, m e\right), M e m_{F}\right)\right\} \leq_{V} \rho_{F}(f, m)$. We define $\operatorname{Reach}_{P}^{F}$ as the least set satisfying:

$$
\begin{aligned}
& \text { [prg] }(P,[]) \in \operatorname{Reach}_{P}^{F} \\
& \text { [fun] } \left.\left(\left(\mu f \cdot \lambda^{l} x \cdot u e_{0}^{l_{0}}, m e\right) \in \operatorname{Reach}_{P}^{F} \wedge \operatorname{Analyzes}_{m}^{F}\left(\mu f \cdot \lambda x \cdot u e_{0}^{l_{0}}, m e\right)\right)\right) \Rightarrow \\
& \left\{\left(u e_{0}{ }^{l_{0}}, m e[f, x \mapsto m]\right),(x, m),(f, m)\right\} \subseteq \operatorname{Reach}_{P}^{F} \\
& \text { [app] }\left(e_{1} @_{l} e_{2}, m e\right) \in \operatorname{Reach}_{P}^{F} \Rightarrow\left\{\left(e_{1}, m e\right),\left(e_{2}, m e\right)\right\} \subseteq \operatorname{Reach}_{P}^{F}
\end{aligned}
$$

\footnotetext{
${ }^{4}$ It is tempting to write " $\Phi_{F}\left((l, m e), a c_{0}\right)$ " in Fig. 3 (thus replacing $l_{2}$ by $l$ ), but then subject reduction for flows would not hold.

${ }^{5}$ Unlike [JWW97, we do not compare analyses with different sets of mementoes.

6 This is somewhat similar to the reachability predicate of [GNN97.
} 
[suc] $\left(\operatorname{succ}^{l} e_{1}, m e\right) \in \operatorname{Reach}_{P}^{F} \Rightarrow\left(e_{1}, m e\right) \in \operatorname{Reach}_{P}^{F}$

[if] $\quad\left(\right.$ ifo $e_{0}$ then $e_{1}$ else $\left.e_{2}, m e\right) \in \operatorname{Reach}_{P}^{F} \Rightarrow$ $\left\{\left(e_{0}, m e\right),\left(e_{1}, m e\right),\left(e_{2}, m e\right)\right\} \subseteq \operatorname{Reach}_{P}^{F}$

Example 6. It is easy to verify that for $u e^{l} \in \operatorname{SubExpr}_{\mathrm{P}_{1}}$ it holds that $\mathcal{C}_{\mathrm{F}_{1}}(, l) m e \neq$ $\perp$ iff $\left(u e^{l}, m e\right) \in \operatorname{Reach}_{\mathrm{P}_{1}}^{\mathrm{F}_{1}}$, and that $\rho_{\mathrm{F}_{1}}(z, m) \neq \perp$ iff $(z, m) \in \operatorname{Reach}_{\mathrm{P}_{1}}^{\mathrm{F}_{1}}$.

Lemma 2. Let $F$ be a flow analysis for $P$ such that $F$ is valid. If $\left(u e^{l}, m e\right) \in$ $\operatorname{Reach}_{P}^{F}$ then (i) $\mathcal{C}_{F}(l, m e) \neq \perp$ and (ii) whenever $(z \mapsto m) \in$ me then $(z, m) \in$ Reach $_{P}^{F}$ holds. Also, if $(z, m) \in$ Reach $_{P}^{F}$ then $\rho_{F}(z, m) \neq \perp$.

\section{$5 \quad$ Translating Types to Flows}

Let a uniform typing $T$ for a program $P$ be given. We now demonstrate how to construct a corresponding flow analysis $F=\mathcal{F}(T)$ such that $F$ is valid and safe. First define $\mathrm{Mem}_{F}$ as $I T_{T}$; note that then an address can serve as a memento environment. Next we define a function $\mathcal{F}_{T}$ that translates from $U T y p_{T}$, that is the union types that can be built using $I T_{T}$ and $U T_{T}$, into FlowSet ${ }_{F}$ :

$$
\begin{gathered}
\mathcal{F}_{T}\left(\bigvee_{i \in I}\left\{q_{i}: t_{i}\right\}\right)= \\
\left\{((\mu f . \lambda x . e, m e), M) \mid \exists i \in I \text { with } M=\operatorname{dom}\left(t_{i}\right):\right. \\
\quad \text { a judgement for } \mu f . \lambda^{l} x . e \text { occurs in } D_{T} \text { with address me } \\
\quad \text { and is justified by [fun] }{ }^{\left(q_{i}: t\right)} \text { where } t \leq \wedge \\
\left.\cup \text { (if } \exists i \text {. such that } q_{i}=q_{\text {int }} \text { then }\{\text { Int }\} \text { else } \emptyset\right)
\end{gathered}
$$

The idea behind the translation is that $\mathcal{F}_{T}(u)$ should contain all the closures that are "sources" of elementary types in $u$; it is easy to trace such closures thanks to the presence of U-tags. The condition $t \leq_{\wedge} t_{i}$ is needed as a "sanity check", quite similar to the "trimming" performed in [Hei95], to guard against the possibility that two unrelated entities in $D_{T}$ incidentally have used the same $\mathrm{U}$-tag $q_{i}$. As the types of $\mathrm{P} \& \mathrm{P}$ do not contain fun-witnesses, their translation has to rely solely on this sanity check (at the cost of precision, cf. Sect. 1).

Lemma 3. The function $\mathcal{F}_{T}$ is monotone.

Definition 3. With $T$ a typing for $P$, the flow analysis $F=\mathcal{F}(T)$ is given by $\left(P, I T_{T}, \mathcal{C}_{F}, \rho_{F}, \Phi_{F}\right)$, where $\mathcal{C}_{F}, \rho_{F}$, and $\Phi_{F}$ are defined below:

$\mathcal{C}_{F}(l, m e)=\mathcal{F}_{T}(u)$ iff $D_{T}$ contains a judgement $A \vdash u e^{l}: u$ with address me

$\rho_{F}(z, m)=\mathcal{F}_{T}(u)$ iff $u=A_{T}(z, m)$

$\Phi_{F}\left(\left(l_{2}, m e\right),\left(\mu f . \lambda x . e_{0}, m e^{\prime}\right)\right)=M$ iff there exists $q$ such that $D_{T}$ contains a judgement for $\mu f . \lambda x . e_{0}$ at $m e^{\prime}$ derived by $[\mathrm{fun}]^{(q: t)}$,

a judgement for $e_{1} @ u_{2}{ }_{2}$ at me derived by $[\mathrm{app}]^{w^{@}}$ where $w^{@}(q)=M$.

Example 7. With terminology as in Examples 2 and 3, it is easy to check that $\mathcal{F}_{\mathrm{T}_{1}}\left(u_{\mathrm{x}}^{\prime}\right)=\left\{v_{\mathrm{x}}^{\prime}\right\}$ and that $\mathcal{F}_{\mathrm{T}_{1}}\left(u_{\mathrm{x}}\right)=\left\{v_{\mathrm{x}}\right\}$, and that $\mathrm{F}_{1}=\mathcal{F}\left(\mathrm{T}_{1}\right)$. 
We have the following result, where the proof that $F$ is valid is by coinduction.

Theorem 1. With $T$ a uniform typing for $P$, for $F=\mathcal{F}(T)$ it holds that

- F is valid and safe

$-\left(u e^{l}, m e\right) \in \operatorname{Reach}_{P}^{F}$ iff $\mathcal{C}_{F}(l, m e) \neq \perp\left(\right.$ for ue $\left.\in \operatorname{SubExpr}_{P}\right)$

$-(z, m) \in \operatorname{Reach}_{P}^{F}$ iff $\rho_{F}(z, m) \neq \perp$

\section{Translating Flows to Types}

Let a flow analysis $F$ for a program $P$ be given, and assume that $F$ is valid and safe. We now demonstrate how to construct a corresponding uniform typing $T=\mathcal{T}(F)$. First we define $I T_{T}$ as $M e m_{F}$ and $U T_{T}$ as $A b s C l o s_{F} \cup\left\{q_{\text {int }}\right\}$. Next we define a function $\mathcal{T}_{F}$ that translates from FlowSet $_{F}$ into UTyp $_{T}$; inspired by $\mathrm{P} \& \mathrm{P}$ (though the setting is somewhat different) we stipulate:

$\mathcal{T}_{F}(V)=\bigvee_{v \in V}\left\{q_{v}: t_{v}\right\}$ where

if $v=$ Int then $q_{v}=q_{\text {int }}$ and $t_{v}=$ int

if $v=(a c, M)$ with $a c=\left(\mu f \cdot \lambda x \cdot e_{0}{ }^{l}, m e\right)$

then $q_{v}=a c$

and $t_{v}=\bigwedge_{m \in M_{0}}\left\{\{m\}: \mathcal{T}_{F}\left(\rho_{F}(x, m)\right) \rightarrow \mathcal{T}_{F}\left(\mathcal{C}_{F}\left(l_{0}, m e[f, x \mapsto m]\right)\right)\right\}$ where $M_{0}=\left\{m \in M \mid\right.$ Analyzes $\left._{m}^{F}(a c)\right\}$.

The above definition determines a unique union type $\mathcal{T}_{F}(V)$, since recursion is "beneath a constructor" and since FlowSet $F$ is finite (ensuring regularity).

Example 8. With terminology as in Examples 2 and 3 it is easy to see - provided that $q_{\mathrm{x}}$ is considered another name for $a c_{\mathrm{x}}$ - first that $\mathcal{T}_{\mathrm{F}_{1}}\left(\left\{v_{\mathrm{x}}^{\prime}\right\}\right)=u_{\mathrm{x}}^{\prime}$, and then that $\mathcal{T}_{\mathrm{F}_{1}}\left(\left\{v_{\mathrm{x}}\right\}\right)=u_{\mathrm{x}}$ since $\mathcal{T}_{\mathrm{F}_{1}}\left(\left\{v_{\mathrm{x}}\right\}\right) \cdot q_{\mathrm{x}}$ can be found as

$$
\begin{aligned}
& \bigwedge\left(\{1\}: \mathcal{T}_{\mathrm{F}_{1}}\left(\rho_{\mathrm{F}_{1}}(\mathrm{x}, 1)\right) \rightarrow \mathcal{T}_{\mathrm{F}_{1}}\left(\mathcal{C}_{\mathrm{F}_{1}}\left(7, m e_{\mathrm{x} 1}\right)\right),\{2\}: \mathcal{T}_{\mathrm{F}_{1}}\left(\rho_{\mathrm{F}_{1}}(\mathrm{x}, 2)\right) \rightarrow \mathcal{T}_{\mathrm{F}_{1}}\left(\mathcal{C}_{\mathrm{F}_{1}}\left(7, m e_{\mathrm{x} 2}\right)\right)\right) \\
= & \bigwedge\left(\{1\}: \mathcal{T}_{\mathrm{F}_{1}}(\{\operatorname{Int}\}) \rightarrow \mathcal{T}_{\mathrm{F}_{1}}(\{\operatorname{Int}\}),\{2\}: \mathcal{T}_{\mathrm{F}_{1}}\left(\left\{v_{\mathrm{x}}^{\prime}\right\}\right) \rightarrow \mathcal{T}_{\mathrm{F}_{1}}\left(\left\{v_{\mathrm{x}}^{\prime}\right\}\right)\right) \\
= & \bigwedge\left(\{1\}: u_{\mathrm{int}} \rightarrow u_{\mathrm{int}},\{2\}: u_{\mathrm{x}}^{\prime} \rightarrow u_{\mathrm{x}}^{\prime}\right) .
\end{aligned}
$$

Note that without the $M$ component in a flow value $(a c, M), v_{\mathrm{x}}$ would equal $v_{\mathrm{x}}^{\prime}$ causing $\mathcal{T}_{\mathrm{F}_{1}}\left(\left\{v_{\mathrm{x}}\right\}\right)$ to be an infinite type (as in $\left.\mathrm{P} \& \mathrm{P}\right)$.

Lemma 4. The function $\mathcal{T}_{F}$ is monotone.

For $z$ and $m$ such that $(z, m) \in \operatorname{Reach}_{P}^{F}$, we define $\mathcal{T}_{F}^{\rho}(z, m)$ as $\mathcal{T}_{F}\left(\rho_{F}(z, m)\right)$ (by Lemma 2 this is well-defined). And for $e=u e^{l}$ and $m e$ such that $(e, m e) \in$ Reach $F$, we construct a judgement $\mathcal{T}_{F}^{J}(e, m e)$ as

$$
\mathcal{T}_{F}^{A}(m e) \vdash e: \mathcal{T}_{F}\left(\mathcal{C}_{F}(l, m e)\right)
$$

where $\mathcal{T}_{F}^{A}(m e)$ is defined recursively by $\mathcal{T}_{F}^{A}([])=[]$ and $\mathcal{T}_{F}^{A}(m e[z \mapsto m])=$ $\mathcal{T}_{F}^{A}(m e)\left[z \mapsto \mathcal{T}_{F}^{\rho}(z, m)\right]$ (by Lemma 2 also this is well-defined). 
Definition 4. With $F$ a flow analysis for $P$, the typing $T=\mathcal{T}(F)$ is given by $\left(P\right.$, Mem $_{F}$, AbsClos $\left._{F} \cup\left\{q_{\mathrm{int}}\right\}, D_{T}\right)$, where $D_{T}$ is defined by stipulating that whenever $(e, m e)$ is in Reach $F$ then $D_{T}$ contains $\mathcal{T}_{F}^{J}(e, m e)$, and that $\mathcal{T}_{F}^{J}\left(e^{\prime}, e^{\prime}\right)$ is a premise of $\mathcal{T}_{F}^{J}(e, m e)$ iff $(e, m e) \in$ Reach $_{P}^{F}$ is among the immediate conditions (cf. the definition of Reach $\left.{ }_{P}^{F}\right)$ for $\left(e^{\prime}, m^{\prime}\right) \in \operatorname{Reach}_{P}^{F}$.

Example 9. It is easy to check that $\mathrm{T}_{1}=\mathcal{T}\left(\mathrm{F}_{1}\right)$, modulo renaming of the U-tags.

Clearly $D_{T}$ is a tree-formed derivation, and $\mathcal{T}_{F}^{J}(e, m e)$ has address $m e$ in $D_{T}$. We must of course also prove that all judgements in $D_{T}$ are in fact derivable from their premises using the inference rules in Fig. 1

Theorem 2. If $F$ is valid and safe then $T=\mathcal{T}(F)$ as constructed by Definition 4 is a uniform typing for $P$. The derivation $D_{T}$ has the following properties:

- if $D_{T}$ contains at address me a judgement for $\mu f . \lambda x . e$, it is derived using $[\text { fun }]^{w^{\lambda}}$ where $w^{\lambda}=\left(a c:\left(\mathcal{T}_{F}\left(\left\{\left(a c, M e m_{F}\right)\right\}\right)\right) . a c\right)$ with ac $=(\mu f . \lambda x . e, m e)$;

- if $D_{T}$ contains at address me a judgement for $e_{1} @ u_{2}{ }_{2}$ with the leftmost premise of the form $A \vdash e_{1}: u_{1}$, then it is derived using [app] ${ }^{w^{@}}$ where for all $q \in \operatorname{dom}\left(u_{1}\right)$ it holds that $w^{@}(q)=\Phi_{F}\left(\left(l_{2}, m e\right), q\right)$.

\section{Round Trips}

Next consider the "round-trip" translations $\mathcal{F} \circ \mathcal{T}$ (from flows to types and back) and $\mathcal{T} \circ \mathcal{F}$ (from types to flows and back). Both roundtrips are idempotent 7 : they act as the identity on "canonical" elements, and otherwise "canonicalize".

Example 10. Exs. 7 and 9 show that $\mathcal{F} \circ \mathcal{T}$ is the identity on $\mathrm{F}_{1}$ and that $\mathcal{T} \circ \mathcal{F}$ is the identity (modulo renaming of U-tags) on $\mathrm{T}_{1}$. In particular $\mathcal{T} \circ \mathcal{F}$ does not necessarily introduce infinite types, thus solving an open problem in $\mathrm{P} \& \mathrm{P}$.

\subsection{Round Trips from the Flow World}

$\mathcal{F} \circ \mathcal{T}$ filters out everything not reachable, and acts as the identity ever after.

Theorem 3. Assume that $F$ is valid and safe for a program $P$, and let $F^{\prime}=$ $\mathcal{F}(\mathcal{T}(F))$. Then $F^{\prime}$ is valid and safe for $P$ with Mem $_{F^{\prime}}=$ Mem $_{F}$, Reach $F_{P}^{\prime}=$ $\operatorname{Reach}_{P}^{F}$, and $\mathcal{C}_{F^{\prime}}(l, m e) \neq \perp$ iff $\left(\mathcal{C}_{F}(l, m e) \neq \perp\right.$ and $\left.\left(u e^{l}, m e\right) \in \operatorname{Reach}_{P}^{F}\right)$ in which case $\mathcal{C}_{F^{\prime}}(l, m e)=\operatorname{filter}_{P}^{F}\left(\mathcal{C}_{F}(l, m e)\right)$ where $\operatorname{filter}_{P}^{F}(V)$ is given by

$\left\{\left(a c, M^{\prime}\right) \mid(a c, M) \in V\right.$ and $\left(\mu f . \lambda x . e_{0}, m_{0}\right) \in$ Reach $_{P}^{F}$ where $a c=\left(\mu f . \lambda x \cdot e_{0}, m e_{0}\right)$ and $M^{\prime}=\left\{m \in M \mid\left(e_{0}, m e_{0}[f, x \mapsto m]\right) \in \operatorname{Reach}_{P}^{F}\right\}$

$\cup($ if Int $\in V$ then $\{$ Int $\}$ else $\emptyset)$.

Finally, if $F^{\prime \prime}=\mathcal{F}\left(\mathcal{T}\left(F^{\prime}\right)\right)$ then $F^{\prime \prime}=F^{\prime}$.

\footnotetext{
${ }^{7}$ However, $\mathcal{T}(\mathcal{F}(\mathcal{T}(F)))=\mathcal{T}(F)$ does in general not hold.
} 
Clearly everything not reachable may be considered "junk". However, simple examples demonstrate that some junk is reachable and is hence not removed by $\mathcal{F} \circ \mathcal{T}$. That our flow/type correspondence can faithfully encode such imprecisions illustrates the power of our framework.

\subsection{Round Trips from the Type World}

The canonical typings are the ones that are strongly consistent:

Definition 5. A typing $T$ is strongly consistent iff for all $u$ that occur in $D_{T}$ and for all $q \in \operatorname{dom}(u)$ with $q \neq q_{\text {int }}$ the following holds: $D_{T}$ contains exactly one judgement derived by an application of [fun $]^{w^{\lambda}}$ with $w^{\lambda}$ taking the form $(q: t)$, and this $t$ satisfies $t \leq_{\wedge}^{c}$ u.q. Here $\leq_{\wedge}^{c}$ is a subrelation of $\leq_{\wedge}$, defined by stipulating that int $\leq_{\wedge}^{c}$ int and that $\bigwedge_{i \in I}\left\{K_{i}: u_{i} \rightarrow u_{i}^{\prime}\right\} \leq_{\wedge}^{c} \bigwedge_{i \in I_{0}}\left\{K_{i}: u_{i} \rightarrow u_{i}^{\prime}\right\}$ iff $I_{0} \subseteq I$.

Theorem 4. Assume that $T$ is a uniform typing for a program $P$, and let $T^{\prime}=$ $\mathcal{T}(\mathcal{F}(T))$. Then $T^{\prime}$ is a uniform typing for $P$ with $I T_{T^{\prime}}=I T_{T}$, and

- $D_{T^{\prime}}$ contains a judgement for $e$ with address ke iff $D_{T}$ contains a judgement for e with address ke (i.e., the two derivations have the same shape);

- $D_{T^{\prime}}$ is strongly consistent;

- if $D_{T}$ is strongly consistent then $D_{T^{\prime}}=D_{T}$ (modulo renaming of U-tags).

Example 11. Let $T$ be the typing 8 of the motivating example put forward in Sect. 1. Then $T$ is not strongly consistent, but $T^{\prime}=\mathcal{T}(\mathcal{F}(T))$ is: the two funwitnesses occurring in $D_{T^{\prime}}$ are of the form $\left(q_{\mathrm{x}}: u_{\mathrm{int}} \rightarrow u_{\mathrm{int}}\right)$ and $\left(q_{\mathrm{y}}: u_{\mathrm{int}} \rightarrow u_{\mathrm{int}}\right)$. Nevertheless, $T^{\prime}$ is still imprecise: both function abstractions are assigned the union type $\bigvee\left(q_{\mathrm{x}}: u_{\mathrm{int}} \rightarrow u_{\mathrm{int}}, q_{\mathrm{y}}: u_{\mathrm{int}} \rightarrow u_{\mathrm{int}}\right)$.

\section{Discussion}

Our flow system follows the lines of N\&N, generalizing some features while omitting others (such as polymorphic splitting [WJ98], left for future work). That it has substantial descriptive power is indicated by the fact that it encompasses both argument-based and call-string based polyvariance. In particular, the flow analysis framework of P\&P can be encoded into our framework. Unlike P\&P, our flow logic has a subject reduction property, inherited from the N\&N approach.

The generality of our type system is less clear. The annotation with tags gives rise to intersection and union types that are not associative, commutative, or idempotent (ACI). This stands in contrast to the ACI types of P\&P, but is similar to the non-ACI intersection and union types of CIL, the intermediate language of an experimental compiler that integrates flow information into the type system [WDMT97|DMTW97. Indeed, a key motivation of this work was to formalize the encoding of various flow analyses in the CIL type system. Developing a translation between the the type system of this paper and CIL is our next goal.

\footnotetext{
${ }^{8}$ We convert it to our framework by substituting $u_{\text {int }}$ for int and by substituting $\bigvee\left(q \bullet: \bigwedge\left(\{\bullet\}: u_{\text {int }} \rightarrow u_{\text {int }}\right)\right)$ for int $\rightarrow$ int.
} 


\section{References}

AC93. R. Amadio and L. Cardelli. Subtyping recursive types. ACM Trans. on Prog. Langs. and Systs., 15(4):575-631, 1993.

Age95. O. Agesen. The cartesian product algorithm. In Proceedings of ECOOP'95, Seventh European Conference on Object-Oriented Programming, vol. 952, pp. 2-26. Springer-Verlag, 1995.

AT00. T. Amtoft and F. Turbak. Faithful translations between polyvariant flows and polymorphic types. Technical Report BUCS-TR-2000-01, Comp. Sci. Dept., Boston Univ., 2000.

Ban97. A. Banerjee. A modular, polyvariant, and type-based closure analysis. In ICFP '97 ICFP97.

DMTW97. A. Dimock, R. Muller, F. Turbak, and J. B. Wells. Strongly typed flowdirected representation transformations. In ICFP '97 [ICFP97], pp. 11-24.

GNN97. K. L. S. Gasser, F. Nielson, and H. R. Nielson. Systematic realisation of control flow analyses for CML. In ICFP '97 [CFP97], pp. 38-51.

Hei95. N. Heintze. Control-flow analysis and type systems. In SAS '95 SAS95, pp. 189-206.

ICFP97. Proc. 1997 Int'l Conf. Functional Programming, 1997.

JW95. S. Jagannathan and S. Weeks. A unified treatment of flow analysis in higher-order languages. In Conf. Rec. 22nd Ann. ACM Symp. Princ. of Prog. Langs., pp. 393-407, 1995.

JWW97. S. Jagannathan, S. Weeks, and A. Wright. Type-directed flow analysis for typed intermediate languages. In Proc. 4th Int'l Static Analysis Symp., vol. 1302 of LNCS. Springer-Verlag, 1997.

NN97. F. Nielson and H. R. Nielson. Infinitary control flow analysis: A collecting semantics for closure analysis. In Conf. Rec. POPL '97: 24th ACM Symp. Princ. of Prog. Langs., pp. 332-345, 1997.

NN99. F. Nielson and H. R. Nielson. Interprocedural control flow analysis. In Proc. European Symp. on Programming, vol. 1576 of LNCS, pp. 20-39. Springer-Verlag, 1999.

PO95. J. Palsberg and P. O'Keefe. A type system equivalent to flow analysis. ACM Trans. on Prog. Langs. and Systs., 17(4):576-599, 1995.

PP98. J. Palsberg and C. Pavlopoulou. From polyvariant flow information to intersection and union types. In Conf. Rec. POPL '98: 25th ACM Symp. Princ. of Prog. Langs., pp. 197-208, 1998. Superseded by [PP99].

PP99. J. Palsberg and C. Pavlopoulou. From polyvariant flow information to intersection and union types. A substantially revised version of [PP98]. Available at http://www.cs.purdue.edu/homes/palsberg/paper /popl98.ps.gz., Feb. 1999.

SAS95. $\quad$ Proc. 2nd Int'l Static Analysis Symp., vol. 983 of LNCS, 1995.

Sch95. D. Schmidt. Natural-semantics-based abstract interpretation. In SAS '95 SAS95, pp. 1-18.

Shi91. O. Shivers. Control Flow Analysis of Higher Order Languages. PhD thesis, Carnegie Mellon University, 1991.

WDMT97. J. B. Wells, A. Dimock, R. Muller, and F. Turbak. A typed intermediate language for flow-directed compilation. In Proc. 7th Int'l Joint Conf. Theory $\&$ Practice of Software Development, pp. 757-771, 1997.

WJ98. A. Wright and S. Jagannathan. Polymorphic splitting: An effective polyvariant flow analysis. ACM Trans. on Prog. Langs. and Systs., 20:166-207, 1998. 